\title{
Learning Through Practical Involvement in the OSS Ecosystem: Experiences from a Masters Assignment
}

\author{
Björn Lundell, Anna Persson and Brian Lings \\ University of Skövde, P.O. Box 408, SE-541 28 SKÖVDE, Sweden \\ \{bjorn.lundell, anna.persson, brian.lings \}@his.se
}

\begin{abstract}
Increased awareness of and interest in Open Source has led to a number of university teaching initiatives, at both national and European level. In this paper we present experiences from a practical assignment designed to give students on an Open Source Masters course an insight into real involvement in Open Source projects. It discusses the motivations for the assignment, and how it was set up and executed. It reports on post facto student feedback, and reflects on a parallel, reduced exercise offered at undergraduate level. We find that the learning experience was both positive and valuable in that it gave real insight into Open Source participation, and also encouraged further participation in Open Source projects by students after the course had completed.
\end{abstract}

\section{Introduction}

This paper relates to a Masters course in Open Source and Distributed Development models. In particular, it focuses on the practical assignment for the course, which was specifically on participation in Open Source (OS) development. The course is a new addition to an existing Masters that has been running since 1990. The course was a natural outcome of the University's growing involvement in OS research, and in particular its partnership in two EU-funded research projects in the area (the FP6 project CALIBRE and the ITEA project COSI).

The course was motivated by the recent growth in interest and activity in OS nationally, in industry and the public sector. Apart from the recognised business opportunities offered by OS (Fitzgerald, 2006), there has been a growing awareness of the opportunities offered for organisational learning and individual skills development through participation in OS projects. Such awareness has led to a number of initiatives in teaching, particularly at the Masters level, at both national and European level (e.g. Fernandes and Machado, 2006; German, 2005; Megías et al., 2005; Özel et al., 2006).

However, the question naturally arises: can one teach about OS participation in a meaningful way in a classroom situation? There is a natural tension between the study of OS as a phenomenon, and OS as a way of contributing practically to community-driven projects. In designing the course this tension was recognised, and it was planned to at least partially address it through a practical assignment.

Please use the following format when citing this chapter.

Lundell, B., Persson, A. and Lings, B., 2007, in IFIP International Federation for Information Processing, Volume 2.34, Open Source Development, Adoption and Innovation, eds. J. Feller, Fitzgerald, B., Scacchi, W., Sillitti. A., (Boston: Springer), pp. 289-294 
The aim of this paper is not to describe the complete course; instead the focus is on the practical assignment that the students carried out during the course.

\section{The Masters Course}

The graduate level course "Open Source and Distributed Development Models" (7.5 ECTS points) was offered at the University of Skövde during the autumn of 2006. The course was an international course, offered in English, and had 12 participants. To attend the course, a bachelor's degree in computer science or a closely related subject was required. The course aims to develop an understanding of open source software (OSS) systems and distributed development models for software systems and applications. Students taking the course are exposed to contemporary issues and industrial practice in OS, and the latest research results related to OS. Key to the course is student interaction with researchers and practitioners actively involved in the OS area, and real exposure to the Open Source ecosystem. The motivation for offering this course came from an observation of a growing student interest in OS and its relevance to industry, and was made possible by ongoing research activities in this area at the university.

Since 1990 the University of Skövde has adopted a profile for its advanced courses which has been strongly influenced by the UK model of postgraduate, research oriented Masters. It has a fairly conventional format, with class-room lectures from researchers and practitioners actively involved in the OS area (both from in-house speakers and invited speakers) and seminars based around research papers. The course has a number of intended learning outcomes (ILOs) against which students are assessed. In particular, in planning the course it was felt necessary that students should learn about the practicalities of what it means to contribute to an OS project. A practical assignment was seen as an effective way to expose students to real OSS ecosystems, and thereby contribute to two specific ILOs:

- Demonstrate knowledge of the major sources of information on OS projects and issues, and an ability to use these sources critically and effectively to report on OS projects and OS issues.

- Rigorously analyse work practices and development models used in Open Source and traditional Distributed Development projects.

The aim of the assignment is for students to get a feeling for how major open source style projects work in action, by contributing. All students had previous experience of using OSS. However, only one had experience of contributing to an OS project.

The assignment had two parts, both practical, which are described in the remainder of this section. In preparation for the assignment, two specific readings had been added to the reading list as a general background on open source projects and their philosophy. The first was "The Cathedral and the Bazaar" by Eric Raymond (Raymond, 1999) and the second the GNU Manifesto by Richard Stallman (Stallman, 2006). 
Experiences from a Masters Assignment

\section{Assignment Part 1: Introduction to Open Source Software in Practice}

The first part was designed to exercise specific and limited aspects of contributing to OS projects, namely: (a) selecting an area to which the student felt it possible to contribute, (b) exposing a contribution to a wide community, and (c) learning tools/processes of a community.

Wikipedia (http://en.wikipedia.org) - a free, extensive, on-line encyclopedia entirely edited by its users - was the chosen medium for this exercise. More specifically, students were asked to make a contribution of content to Wikipedia. This had the advantage of giving a feel for open contribution without the overhead necessary to engage meaningfully with a software development community. Other advantages in choosing Wikipedia for the first part of the assignment include: (a) it is an OS project that a majority of the students are already familiar with; many of them use Wikipedia in searching for information on a regular basis, (b) it is easy to make a contribution as no special permissions or development tools are required, and (c) one does not need to wait for approval of a contribution, rather it is immediately visible. Only two requirements were expressed for the contribution: (1) it should include an English text of 150-200 words, and (2) it should include a picture.

\section{Assignment Part 2: Open Source Development}

The aim of the second part of the assignment was for students to gain an understanding of OSS development by taking part in a currently active OS project. We argue that a student's understanding of OS development will be considerably improved with practical experience of being part of a real project. It is hard to give insight into what an OS project really means unless it is grounded in experience.

Students were asked to make a contribution to one project (corresponding to about one week full time), and meanwhile examine the development work within the project. They could pick any project of their choice (Sourceforge and Tigris were recommended as starting points for exploration). A contribution could relate to code (new feature, bug fix, patch, etc), software testing, design specification, manual content, or web content.

Some interaction with the project was expected, and a genuine effort made by the student to make the contribution openly visible in the project. Ideally, a contribution would be accepted by the project and incorporated in the code/documentation base. However, it was recognised that in some projects this process may take longer than the course period and so was not a requirement.

In the end of the assignment period, students had to write an essay (2500 words plus appendices) of their contribution and the general development work within the chosen project, with a focus on the distributed perspective. In the text, they were required to position their experience with respect to the research literature. As a minimum, it was expected that each student would use at least two reviewed publications when relating their own observations and argument to the existing body of literature. 


\section{Experiences from the Assignment}

This section describes student experience of the practical assignment as expressed in their final reports and a debriefing seminar which was held after completion of the assignment.

\section{Assignment Part 1: Introduction to Open Source Software in Practice}

All of the students used Wikipedia almost daily. Despite this none of them had made a contribution to the encyclopaedia before. The students found the material that Wikipedia provides about how to edit articles straightforward and rather easy to understand.

The students thought that the hardest part of the assignment was to find an article that they felt comfortable to contribute to. They perceived that the openness of Wikipedia encourages a lot of intelligent people to write on most topics, so most of the articles they looked into already had good coverage.

When finally finding an article, they put a lot of effort into making their text as good and correct as possible, knowing that many users read the website and that incorrect or badly written text will very likely be edited or even removed. They found it demanding to adopt the same style of writing as the rest of the article. Preparing for image upload held surprises. Firstly, no conflicting licenses can be incorporated into Wikipedia, so they were required to verify that the license on the uploaded material was appropriate for Wikipedia. Further, the whole upload section of Wikipedia has a flat hierarchy; all files are put in the same place in the structure, and referenced only by the filename entered for it when uploading.

In summary, students felt that contributing to Wikipedia was a positive experience and all of them claimed that they will make further contributions to Wikipedia in the future. They really appreciated the openness of the system which puts editing any content on the whole site only one click away.

\section{Assignment 2: Open Source Development}

Initially, some students thought that this assignment seemed very hard to carry out. The reason was that they assumed "contribute to an OS project" meant "contributing code", which is a common misconception. On the contrary, there is actually a great need for contributions other than code - such as design documents, manual content, etc. When this was explained to the students they felt relieved as they lacked confidence in their own programming skills so were unsure of their ability to make a high-quality code contribution. However, five students did choose to contribute code, whereas the others decided to submit bug reports, documentation and desktop themes. One student wanted to concentrate only on submitting feature requests, but this was not considered appropriate as it lacked real engagement in the project. Examples of projects attracting student involvement include Premake, Robocode, $\mathrm{KDE}$ and Vim. 
One thing that surprised some of the students was how well-organised their selected projects were. Their previous view of OS projects was of more or less chaotic anarchistic communities. What many students began to realise whilst undertaking the assignment was that many OS projects actually are quite wellstructured with explicit work processes and clear roles.

The experience of most students was that their commitment to a project was well received. In the final report, one of the students wrote:

"I don't know if I should say that I was surprised by the kind treatment I got, but I was definitely surprised how glad they were to let me join the development team. The kind of welcome I got definitely made me more interested in joining open source projects if I find that something needs fixing."

Wishing to continue involvement in OS projects was a recurring theme. For example, one student wrote:

"I think all-in-all it was a good experience to make a real contribution to a real open source project. The open source phenomenon will surely stay for a long time to come and it is only positive to have gained a more direct understanding of how these work and function. In the future I think I will be much more inclined to get my feet wet in various open source projects."

In a similar vein, another student wrote:

"It is highly probable that I will contribute to other existing open source projects in the future, and it might also be the case that I will start one of my own to realize an idea that I have."

One unanticipated problem occurred related to a student employed by a large company. The student got into trouble in making his contribution as the company he works for does not allow employees to make source code or any other contribution to any open source project without written authorization. Due to bureaucratic processes he had not received such authorization before the deadline for the assignment. However, he plans to submit his contribution to the project when such authorisation is received.

\section{Discussion and Conclusions}

Without any previous experience of the Open Source community, many students think that it is very hard to take part in Open Source projects. They think they have to be a well skilled programmer or technician to do it. It is important to change such perceptions.

One may speculate over the extent to which an assignment like this, in which students are forced to make a contribution, genuinely represents a learning experience with respect to Open Source participation. Might it contrast negatively with a regular voluntary contribution without any extra agenda? In mitigation, several points can be made. Firstly, the students chose the course from many options 
in the Masters programme; in fact it was amongst the most popular courses. Secondly, there was little restriction on the projects which could be chosen for making a contribution, and significant calendar time was made available for this. Thirdly, acceptance of contributions by the Open Source community was not a requirement for passing the assignment, only that a student must make a serious effort to contribute, supplemented by reported experiences and documentation of and reflections on genuine interaction with the project.

As we have experienced in this assignment, it may be a problem for people employed at a company to participate in a course like this. Some companies have an organisational policy in order to prevent making the company visible via Open Source contributions. However, it is clear that many companies value interaction with Open Source projects, and such courses are likely to attract company-based students. It is certainly worth our while to attempt to resolve such potential issues. It should also be noted that Scandinavia has a high degree of contributors to OS projects and the typically flat hierarchical structures in Scandinavian organisations may very well be an important background factor which reinforces this.

\section{References}

Fernandes, J. M. and Machado, R. J. (2006) A Two-Year Software Engineering M.Sc. Degree Designed Under the Bologna Declaration Principles, In Proceedings of the International Conference on Software Engineering Advances (ICSEA'06), IEEE Computer Society, p. 1, 2006.

Fitzgerald, B. (2006) The Transformation of Open Source Software, MISQ, Vol. 30, No. 3, pp. 587-598.

German, D. (2005) Experiences teaching a graduate course in Open Source Software Engineering, In Scotto, M. and Succi, G. (Eds.) Proceedings of the First International Conference on Open Source Systems, Genova, Italy, 11-15 July 2005, pp. 326-328, $<$ oss2005.case.unibz.it/Papers/OES/ES1.pdf $>$.

Megías, D., Serra, J. and Macau, R. (2005) An International Master Programme in Free Software in the European Higher Education Space, In Scotto, M. and Succi, G. (Eds.) Proceedings of the First International Conference on Open Source Systems, Genova, Italy, 11-15 July 2005, pp. 349-352, <oss2005.case.unibz.it/Papers/OES/EK3.pdf>.

Raymond, E.S. (1999) The Cathedral \& the Bazaar, O'Reilly. hardcover ISBN 1-56592-7249, October 1999.

Stallman, R. (2006) The GNU Manifesto, <www.gnu.org/gnu/manifesto.html> [Accessed 13 December, 2006].

Özel, B., Gencer, M. and Stephenson, C. (2006) An MSc Programme in Open Source Information Systems, In Özel, B., Burak Çilingir, C., and Erkan, K. (Eds.) Towards Open Source Adoption: Educational, Public, Legal, and Usability Practices - OSS 2006 tOSSad workshop proceedings, Como, Italy, June 10, 2006, TÜ_TAK (The Scientific \& Technological Research Council of Turkey), TÜ_TAK MAM MAtbaasi, 41470, Kocaeli, Turkey. 